\title{
AUTOMORPHISM ORBITS OF FINITE GROUPS
}

\author{
THOMAS J. LAFFEY and DESMOND MacHALE
}

(Received 1 February 1984; revised 12 November 1984)

Communicated by D. E. Taylor

\begin{abstract}
Let $G$ be a finite group and let $\operatorname{Aut}(G)$ be its automorphism group. Then $G$ is called a $k$-orbit group if $G$ has $k$ orbits (equivalence classes) under the action of $\operatorname{Aut}(G)$. (For $g, h \in G$, we have $g \sim h$ if $g^{\alpha}=h$ for some $\alpha \in \operatorname{Aut}(G)$.) It is shown that if $G$ is a $k$-orbit group, then $k \leqslant|G| / p+1$, where $p$ is the least prime dividing the order of $G$. The 3-orbit groups which are not of prime-power order are classified. It is shown that $A_{5}$ is the only insoluble 4-orbit group, and a structure theorem is proved about soluble 4-orbit groups.
\end{abstract}

1980 Mathematics subject classification (Amer. Math. Soc.): $20 \mathrm{~F} \mathrm{28;} \mathrm{secondary} 20 \mathrm{E} 36$.

Let $G$ be a finite group and let $\operatorname{Aut}(G)$ be its automorphism group. Then $G$ is partitioned into equivalence classes under the action of $\operatorname{Aut}(G)$-we say that $g, h$ are equivalent if $g^{\alpha}=h$ for some $\alpha \in \operatorname{Aut}(G)$. The equivalence classes are called automorphism orbits. We call $G$ a $k$-orbit group if it has $k$ automorphism orbits. The identity constitutes the only 1-orbit group, and it is easy to see that the (finite) 2-orbit groups are precisely the elementary abelian groups of prime-power order. In this paper, we prove that if $G$ is a $k$-orbit group, then $k \leqslant 1+|G| / p$ where $p$ is the least prime divisor of $|G|$. We also completely classify the 3-orbit groups which are not of prime-power order and the insoluble 4-orbit groups. We also prove a structure theorem about 4-orbit groups which are not of prime-power order.

Notation. The notation is standard (compare Huppert [1]) with the following additions: $\pi(G)$ denotes the set of prime divisors of $|G|$, Syl $_{p}(G)$ denotes the set of Sylow $p$-subgroups of $G$, and $N$ char $G$ denotes that $N$ is a characteristic subgroup of $G$.

(C) 1986 Australian Mathematical Society $0263-6115 / 86 \$ A 2.00+0.00$ 
We begin with

THEOREM 1. Let $G$ be a finite non-abelian group and let $p$ be the least prime dividing $|G|$. Then $G$ has at most $|G| / p$ automorphism orbits.

Proof. Let $r$ be the number of conjugacy classes of $G$ and $k$ the number of automorphism orbits. Then $k \leqslant r$. Also $G$ has $r$ irreducible complex characters of which $\left|G / G^{\prime}\right|$ are linear and the rest have degree at least $p$. Hence

$$
|G| \geqslant\left|G / G^{\prime}\right|+\left(r-\left|G / G^{\prime}\right|\right) p^{2}
$$

(since the squares of the degrees of the characters all equal $|G|)$. Hence

$$
r \leqslant \frac{1}{p^{2}}\left[|G|+\left(p^{2}-1\right)\left|G / G^{\prime}\right|\right] .
$$

If $\left|G^{\prime}\right|>p$, then $r \leqslant p^{-2}[|G|+(p-1)|G|]=|G| / p$, which proves the result. Hence we may assume that $\left|G^{\prime}\right|=p$. In particular, $G^{\prime} \leqslant Z(G)$. If $A$ is an abelian direct factor of $G$, say $G=A \times B$, then $G$ has at most $|A| m$ automorphism orbits, where $m$ is the number of automorphism orbits of $B$. Hence, by induction, we may assume that $G$ has no abelian direct factors, and hence that $G$ is indecomposable.

So we now have: $G$ is an indecomposable $p$-group, and $\left|G^{\prime}\right|=p$. Let $G / G^{\prime}$ have type $\left(r_{1}, \ldots, r_{m}\right)$, i.e. let $G / G^{\prime}$ be a direct product of $r_{1}$ cyclic groups of order $p, r_{2}$ of order $p^{2}, \ldots, r_{m}$ of order $p^{m}$. Let $Z_{i}=\Omega_{i}(Z(G))=\left\{z \in Z(G) \mid z^{p^{i}}\right.$ $=1\}$. Theorem 1 of Sanders [2] states that the group $C(G)$ of central automorphisms of $G$ has order $\prod_{i=1}^{m}\left|Z_{i}\right|^{r_{i}}$. Hence $|C(G)| \geqslant \prod_{i=1}^{m}\left|Z_{1}\right|^{r_{i}}=\left|Z_{1}\right|^{d}$, where $d=r_{1}+\cdots+r_{m}$ is the minimal number of generators of $G / G^{\prime}$ and hence of $G$.

We call an element $g \in G$ small if

$$
\left|\left\{g^{\sigma} \mid \boldsymbol{\sigma} \in C(G)\right\}\right|<\left|Z_{1}\right|,
$$

and we call $g$ nearly small if

$$
\left|\left\{g^{\sigma} \mid \sigma \in C(G)\right\}\right| \leqslant\left|Z_{1}\right| .
$$

Let $S$ be the subgroup generated by the small elements. Suppose first that $S=G$. Let $\left\{s_{1}, \ldots, s_{d}\right\}$ be a set of small elements which generate $G$. Let $b$ be the $d$-tuple $\left(s_{1}, \ldots, s_{d}\right)$ and, for $\sigma \in C(G)$, let $b^{\sigma}=\left(s_{1}^{\sigma}, \ldots, s_{d}^{\sigma}\right)$. Note that for $\sigma$, $\tau \in C(G)$, we have $b^{\sigma}=b^{\tau}$ if and only if $\sigma \tau^{-1}$ fixes each one of $s_{1}, \ldots, s_{d}$, and thus if and only if $\sigma=\tau$, since $\left\{s_{1}, \ldots, s_{d}\right\}$ generates $G$. Hence $\left|\left\{b^{\sigma} \mid \sigma \in C(G)\right\}\right|$ $=|C(G)|$. However, since $s_{i}$ is small, $\left|\left\{s_{i}^{\sigma} \mid \sigma \in C(G)\right\}\right|<\left|Z_{1}\right|$, and hence $\mid\left\{b^{\sigma} \mid \sigma\right.$ $\in C(G)\}\left.|<| Z_{1}\right|^{d}$. This contradicts Sanders' Theorem 1 [2]. Hence $S \neq G$. 
Suppose that $\left|Z_{1}\right| \geqslant p^{2}$. Then we have

$$
\begin{aligned}
k & \leqslant|Z(G)|+\frac{1}{p}|S-Z(G)|+\frac{1}{p^{2}}|G-S| \\
& \leqslant|G|\left[\frac{1}{p^{2}}+\frac{1}{p} \cdot \frac{p-1}{p^{2}}+\frac{p-1}{p^{3}}\right] \\
& =|G|\left[\frac{3 p-2}{p^{3}}\right] \\
& \leqslant|G| / p .
\end{aligned}
$$

Hence we may assume that $\left|Z_{1}\right|=p$, and thus that $Z(G)$ is cyclic.

Suppose next that $G / G^{\prime}$ is not elementary abelian. Let $T$ be the subgroup generated by the nearly small elements of $G$. Arguing as above, we find that $|C(G)| \leqslant\left|Z_{1}\right|^{d}$, and this contradicts Sanders' theorem unless $Z(G)=Z_{1}$. Hence $|Z(G)|>p$ implies that $T \neq G$, and then we obtain as above, that

$$
k \leqslant|Z(G)|+\frac{1}{p}|T-Z(G)|+\frac{1}{p^{2}}|G-T| \leqslant|G| / p .
$$

Hence we may assume that $|Z(G)|=p$. But $\left|G^{\prime}\right|=p$ implies that $[x, y]^{p}=1$ for all $x, y \in G$, and thus that $\left[x, y^{p}\right]=1$, whence $y^{p} \in Z(G)$. Hence $\Phi(G) \leqslant$ $Z(G)=G^{\prime}$, and $G / G^{\prime}$ is elementary abelian.

So the proof is reduced to a consideration of the following situation: $G / G^{\prime}$ is elementary abelian and $Z(G)$ is cyclic. Suppose that $M$ is a maximal subgroup of $G$ and that $G=M Z(G)$. Let $\sigma \in \operatorname{Aut}(M)$ and let $\sigma_{0} \in \operatorname{Aut}(Z(G)$ ) be such that $\left.\sigma_{0}\right|_{Z(G) \cap M}=\left.\sigma\right|_{Z(G) \cap M}$. Extend $\sigma_{0}$ to $G$ by setting $\sigma_{0}(m)=\sigma(m)$ for all $m \in M$, and by making $\sigma_{0}$ multiplicative. Then $\sigma_{0} \in \operatorname{Aut}(G)$, and so the theorem holds for $G$ if it holds for $M$. Hence we may assume that $M=G$, and thus that $Z(G)=\Phi(G)$ has order $p$. But then $G$ is an extraspecial $p$-group. So $\operatorname{Aut}(G)$ is known (Winter [4]). Suppose $|G|=p^{2 n+1}$. Then $Z(G)$ is composed of two automorphism orbits (by the Theorem of [4]), and each $g \in G \backslash Z(G)$ is conjugate to $g z$ for all $z \in Z(G)$. Hence the number of automorphism orbits of $G$ is at most $2+p^{-1}\left(p^{2 n+1}-p\right)=p^{2 n}+1$ with equality if and only if elements in distinct cosets of $Z(G)$ belong to distinct automorphism orbits. However, by [4, (3c), page 161], the automorphisms of $G$ which act trivially on $G / Z(G)$ are precisely the group of inner automorphisms. Since $G$ has an outer automorphism we conclude that there exist $g_{1}, g_{2} \in G$ such that $g_{1} Z(G) \neq g_{2} Z(G)$, but such that $g_{1}, g_{2}$ lie in the same automorphism orbit. (We remark here that $\operatorname{Aut}(G)$ does not transitively permute the non-trivial elements of $G / Z(G)$ in the case where $G$ has exponent $p^{2}(p>2)$ by the Theorem of [4].) Hence $G$ has at most $p^{2 n}=|G| / p$ automorphism orbits, and the proof is complete. 
Corollary. Let $G \neq 1$ be a finite group. Then $G$ has at most $1+|G| / p$ automorphism orbits, where $p$ is the least prime dividing $|G|$.

Proof. Using Theorem 1, we may assume that $G$ is abelian. Thus $G$ is the direct product of cyclic groups of prime power orders. Since the cyclic group $C\left(q^{k}\right)$ of prime power order $q^{k}$ has exactly $k+1$ automorphism orbits, and since the direct product of $r$ copies of $C\left(q^{k}\right)$ also has exactly $k+1$ automorphism orbits, the result follows immediately except in the case $G=\mathbf{Z}_{2} \times \mathbf{Z}_{4}$. But in this case, $G$ has only 4 automorphism orbits. This completes the proof.

Remark. We note that the bound in Theorem 1 is attained by the dihedral group of order 8 .

We now consider 3-orbit groups.

THEOREM 2. Let $G$ be a finite group which is not of prime-power order. The following are equivalent:

(1) $G$ is a 3-orbit group;

(2) $|G|=p^{n} q$, and $G$ has a normal elementary abelian Sylow p-subgroup $P$, for some primes $p, q$, and for some integer $n \geqslant 1$. Furthermore, $p$ is a primitive root $\bmod q$ (i.e. $q-1$ is the least natural number $e$ with $\left.p^{e} \equiv 1 \bmod q\right)$. Let $Q$ be a Sylow q-subgroup of $G$. Then $P$, regarded as a $\mathrm{GF}(p)[Q]-$ module, is a direct sum of $t \geqslant 1$ copies of the (unique) irreducible $\mathrm{GF}(p)[Q]-$ module of dimension $q-1$. In particular $|P|=p^{t(q-1)}$.

Proof. (1) Assume that $G$ is a 3-orbit group and that $G$ is not of prime-power order. Then $|G|=p^{a} q^{b}$ for some primes $p, q$ and integers $a \geqslant 1, b \geqslant 1$. So $G$ is soluble. We may thus assume that $O_{p}(G) \neq 1$. Since $O_{p}(G)$ char $G$, and since $G$ is a 3-orbit group, we thus find that $P=O_{p}(G)$ is a Sylow p-subgroup of $G$. Also, since $\Omega_{1}\left(Z(P)\right.$ ) char $G, P$ is elementary abelian. Let $Q \in \operatorname{Syl}_{q}(G)$. Since $G$ is a 3-orbit group, it has no element of order $p q$. Hence $Q$ acts fixed-point-freely on $P$, so that $Q$ is cyclic, or $q=2$ and $Q$ is (generalized) quaternion [1, $\mathrm{V}(8.15)]$. Since $Q$ must have exponent $q$, we thus obtain $|Q|=q$.

We now regard $P$ as a $\mathrm{GF}(p)[Q]$-module. We write the operation in $P$ as addition and the action of $Q$ (by conjugation) as multiplication. By Maschke's theorem, $P=P_{1} \oplus \cdots \oplus P_{r}$, where the $P_{i}$ are irreducible $\mathrm{GF}(p)[Q]$-modules. Also by Huppert [1, II(3.10), page 166], $\left|P_{i}\right|=p^{e}$, where $e$ is the order of $p \bmod q$ (i.e. $e$ is the least natural number with $p^{e} \equiv 1 \bmod q$ ). If $Q=\langle\alpha\rangle$, we may choose a basis for $P_{i}$ so that $\alpha$ is represented by the companion matrix of its 
minimal polynomial $m_{i}(\lambda)$ on $P_{i}$. Hence $P_{i}$ is determined up to $\operatorname{GF}(p)\{Q\}$ isomorphism by the minimal polynomial $m_{i}(\lambda)$ of $\alpha$ on $P_{i}$. We now claim that $P_{1}, \ldots, P_{r}$ are all isomorphic modules. For suppose that $P_{1}$ is not isomorphic to $P_{2}$. Then $m_{1}(\lambda) \neq m_{2}(\lambda)$. Let $0 \neq u_{i} \in P_{i}(i=1,2)$. Since $G$ is a 3-orbit group, there exists $\sigma \in \operatorname{Aut}(G)$ with $u_{1}^{\sigma}=u_{1}+u_{2}$. Now $\alpha^{\sigma^{-1}}=\alpha^{k} w$ for some $w \in P$, and for some $k \geqslant 1$ with $(k, q)=1$. Let $g(\lambda)$ be the minimal polynomial of $\alpha^{k}$ on $P_{1}$. Note that $\operatorname{deg} g=p^{e}$. Consider

$$
\begin{aligned}
u_{1}^{\sigma} g(\alpha) & =u_{1}^{\sigma}\left(g\left(\alpha^{\sigma^{-1}}\right)\right)^{\sigma} \\
& =u_{1}^{\sigma}\left(g\left(\alpha^{k}\right)\right)^{\sigma} \quad \text { (since } P \text { is abelian) } \\
& =\left[u_{1} g\left(\alpha^{k}\right)\right]^{\sigma}=0 .
\end{aligned}
$$

Hence $\left(u_{1}+u_{2}\right) g(\alpha)=0$. But the minimal polynomial of $\alpha$ on $u_{1}+u_{2}$ is $m_{1}(\lambda) m_{2}(\lambda)$ (since $m_{1} \neq m_{2}$ implies $\left(m_{1}, m_{2}\right)=1$ ). Since $\operatorname{deg} g=\operatorname{deg} m_{i}$, we have a contradiction. So all the $P_{i}$ are isomorphic $\mathrm{GF}(p)[Q]$-modules.

Next, for any $i$ with $(i, q)=1$, there exists $\tau \in \operatorname{Aut}(G)$ with $\alpha^{\tau}=\alpha^{i}$. Let $0 \neq u \in P$. Then the minimal polynomial $m(\alpha)$ such that $u m(\alpha)=0$ is also the minimal polynomial of $\alpha$ on $P$. But

$$
0=u m(\alpha)=[u m(\alpha)]^{\tau}=u^{\tau} m\left(\alpha^{\tau}\right)=u^{\tau} m\left(\alpha^{i}\right) \quad \text { (since } P \text { is abelian) } .
$$

Hence $m\left(\alpha^{i}\right)=0$, and thus $m(\lambda)$ divides $m\left(\lambda^{i}\right)(i=1,2, \ldots, q-1)$. Hence, if $w$ is a root of $m(x)$ in the algebraic closure of $\operatorname{GF}(p)$, so also is $w^{i}$. Therefore $m(\lambda)$ is divisible by the cyclotomic polynomial $\Phi_{q-1}(\lambda)$. Hence $e \geqslant q-1$, so that $e=q-1$, and the result follows.

(2) Assume that $G$ satisfies (2). Then $Q$ has no fixed point on $P$, so $G$ has elements of order $1, p, q$ only. We first show that any two elements of order $p$ are conjugate. Note that $P$ is a homogeneous $\operatorname{GF}(p)[Q]$-module, so if $0 \neq u \in P$, then $P_{0}=\{u f(\alpha) \mid f(x) \in \mathrm{GF}(p)[x]\}$ is an irreducible $\mathrm{GF}(p)[Q]$-submodule of order $p^{q-1}$ (using the same notation as above). Let $0 \neq v \in P$ and let $P_{1}=$ $\{v f(\alpha) \mid f(x) \in \mathrm{GF}(p)[x]\}$. If $P_{0}=P_{1}$, then we can write $P=P_{0} \oplus P_{2}$ as $\operatorname{GF}(p)[Q]$-modules. But then a routine calculation shows that the map $\sigma$ defined by $\alpha^{\sigma}=\alpha, u^{\sigma}=v$, and $w^{\sigma}=w\left(w \in P_{2}\right)$ extends to an automorphism of $G$.

If $P_{0} \neq P_{1}$, then we may write $P=P_{0} \oplus P_{1} \oplus P_{2}$ as $\mathrm{GF}(p)[Q]$-modules. Again a routine calculation shows that the map $\tau$ defined by $\alpha^{\tau}=\alpha, u^{\tau}=v, v^{\tau}=u$, and $w^{\top}=w\left(w \in P_{2}\right)$ extends to an automorphism $\tau$ of $G$.

We must now show that the elements of order $q$ form a single orbit under Aut $(G)$. Since $P$ transitively permutes the Sylow $q$-subgroups it suffices, by Sylow's theorem, to show that, for all $i$ with $1 \leqslant i \leqslant q-1$, there exists $\theta \in$ $\operatorname{Aut}(G)$ with $\alpha^{\theta}=\alpha^{i}$. 
Let $P=P_{1} \oplus \cdots \oplus P_{t}$ as irreducible $\mathrm{GF}(p)[Q]$-modules and let $0 \neq u_{j} \in P_{j}$. Then each $u \in P_{j}$ is uniquely expressible as $u_{j} f(\alpha)$ for some $f(\lambda) \in \operatorname{GF}(p)[\lambda]$ with $\operatorname{deg} f<q-1$. Define a map $\theta$ by $\alpha^{\theta}=\alpha^{i}, u_{j}^{\theta}=u_{j}$, and $\left(u_{j} f(\alpha)\right)^{\theta}=u_{j} f\left(\alpha^{i}\right)$.

Note that if $0 \neq u \in P$ is such that $u g(\alpha)=0$ for some $g(x) \in \operatorname{GF}(p)[x]$, then $\Phi_{q-1}(\lambda)$ divides $g(\lambda)$, and hence it also divides $g\left(\lambda^{i}\right)$. So $u g\left(\alpha^{i}\right)=0$. This proves that the natural extension of $\theta$ to $P$ is well defined. Thus, by a routine calculation, we see that $\theta$ extends to an automorphism of $G$. This proves that $G$ is a 3-orbit group, and so the proof of the theorem is complete.

We next consider 4-orbit groups.

THEOREM 3. Let $G$ be an insoluble 4-orbit group. Then $G \cong A_{5}$.

Proof. Since $G$ is insoluble, it follows that $|\pi(G)| \geqslant 3$, and hence, since $G$ is a 4-orbit group, that $|\pi(G)|=3$. By the Feit-Thompson theorem, $|G|$ is even. So we may write $\pi(G)=\{2, p, r\}$. Since $G$ is a 4-orbit group, the only possible orders for elements of $G$ are 1, 2, p, $r$. In particular, $G$ has an elementary abelian Sylow 2-subgroup. Let $N$ be a minimal characteristic subgroup of $G$. Then $N$ is the direct product of isomorphic simple groups. Also $G / N$ is at most a 3-orbit group. Hence $G / N$ is soluble, and thus $N$ is not soluble. So $N$ is a 4-orbit group. Since $N$ char $G$, and since $G$ is a 4-orbit group, we must have $N=G$. Since all elements of $G-\{1\}$ have prime order, $N$ is simple. Hence $G$ is simple.

By Walter's classification theorem [3], $G$ is one of the following groups:

(1) $\operatorname{PSL}_{2}(q), q \equiv \pm 3 \bmod 8$;

(2) $\mathrm{SL}_{2}\left(2^{n}\right)$, for some $n \geqslant 2$;

(3) a group of Ree type;

(4) the small Janko group $J_{1}$. For $q$ odd, $\operatorname{PSL}_{2}(q)$ has cyclic subgroups of order $(q \pm 1) / 2$, and thus, since $|\pi(G)|=3$, we must have $(q \pm 1) / 2=2$ in case (1). Thus $q=5$ and $G \cong A_{5}$. Again $\operatorname{SL}_{2}\left(2^{n}\right)$ has cyclic subgroups of order $2^{n} \pm 1$, and so again, since $2^{2 n}-1 \equiv 0 \bmod 3$, we have $2^{n}-1=3$. This leads in case (2) to $n=4$ and $G=\mathrm{SL}_{2}(4) \cong A_{5}$. Groups which satisfy (3) or (4) have elements of nonprime order, and also their orders are divisible by more than three primes. So (3) and (4) are impossible for $G$. This proves the theorem.

We now consider the soluble case.

THEOREM 4. Let $G$ be a finite soluble 4-orbit group which is not of prime-power order. Then $|G|=p^{a} q^{b}$, and $G$ has a normal Sylow $p$-subgroup $P$ for some primes $p$, $q$. Let $Q$ be a Sylow $q$-subgroup of $G$. Then one of the following holds:

(1) $Q$ acts fixed-point-freelyy on $P,|Q|=q$, and $P$ is a 2-orbit or 3-orbit group;

(2) $P$ is elementary abelian, and $Q$ is cyclic of order $q^{2}$ or quaternion of order 8 ;

(3) $G=P \times Q$, where $P, Q$ are elementary abelian. 
Proof. Let $p \in \pi(G)$ be such that $O_{p}(G) \neq 1$. Suppose first that $|\pi(G)|=3$. Let $\pi(G)=\{p, q, r\}$ and suppose that $O_{q}\left(G / O_{p}(G)\right) \neq 1$. Let $Q \in \operatorname{Syl}_{q}(G)$, and let $R \in \operatorname{Syl}_{r}(G)$. Let $1 \neq x \in R$ and let $N=Q O_{p}(G)$. Since the only possible orders for elements of $G$ are $1, p, q, r$, and since $N \triangleleft G$, it follows that $x$ acts fixed-point-freely on $N$. So, by Thompson's theorem on fixed-point-free automorphisms of prime order [1, $\mathrm{V}(8.14)], N$ is nilpotent. But then $G$ has an element of order $p q$. This is a contradiction. Hence $|\pi(G)|=2$.

Let $\pi(G)=\{p, q\}$, let $P \in \operatorname{Syl}_{p}(G)$, and let $Q \in \operatorname{Syl}_{q}(G)$. Suppose that $P$ is not normal in $G$. Then since $\Omega_{1}\left(Z\left(O_{p}(G)\right)\right)$ is characteristic in $G$, and since $G$ is a 4-orbit group, $O_{p}(G)$ is elementary abelian. Furthermore, $Q O_{p}(G)$ and $G / O_{p}(G)$ are 3-orbit groups. By Theorem 2 , firstly $Q$ is cyclic of order $q$, and secondly $Q$ is elementary abelian of order $q^{(p-1)}$ (for some $t \geqslant 1$ ) and $\left|P / O_{p}(G)\right|=p$. Hence $t=p-1=1$, so that $p=2$ and $G / O_{p}(G)$ is dihedral of order $2 q$. The only possible orders for the elements in the four automorphism orbits of $G$ are $1,2, q$, 2 or $1,2, q, 4$. The first possibility implies that $P$ is abelian and hence that $P \leqslant C_{G}\left(O_{p}(G)\right)=O_{p}(G)$. Hence $P$ has exponent 4 , and each element of $P-$ $O_{p}(G)$ has order 4. Now $Q O_{p}(G)$ is characteristic in $G$, and by the Frattini argument, we have $G=O_{p}(G) N_{G}(Q)$. Hence $N_{G}(Q)$ contains a 2-element $\beta \notin$ $O_{2}(G)$. Let $Q=\langle\alpha\rangle$. Then $\left[\alpha, \beta^{2}\right] \in Q$, and $1 \neq \beta^{2} \in O_{2}(G)$. Hence $\left[\alpha, \beta^{2}\right]=1$, and $\alpha \beta^{2}$ is an element of order $2 q$. This is a contradiction. So $P$ is normal in $G$, as required.

Next, if $P$ is not elementary abelian, then $P$ is a 3-orbit group. Hence every element outside $P$ has order $q$ and acts fixed-point-freely on $P$. Thus $|Q|=q$. Suppose then that $P$ is elementary abelian. If the automorphism orbits of $G$ are represented by elements of orders $1, p, p, q$, then again conclusion (1) holds. Suppose these orders are $1, p, q, q$ or $1, p, q, q^{2}$. Then $Q$ acts fixed-point-freely on $P$, so either $Q$ is cyclic of order $q$ or $q^{2}$, or $Q$ is a (generalized) quaternion group. Since $Q$ has exponent at most $q^{2}$, this implies that if $Q$ is non-cyclic, then $Q$ must be quaternion of order 8 .

The only remaining possibility is that $G$ has elements of orders $1, p, q, p q$. Then $Q$ is elementary abelian. For each maximal subgroup $A$ of $Q$, let $C_{A}$ be the centralizer in $P$ of $A$. If $O_{q}(G) \neq 1$, then $O_{p}(G) \times O_{q}(G)$ is a characteristic 4-orbit subgroup of $G$. So it equals $G$, and (3) holds.

Suppose then that $O_{q}(G)=1$, so that $Q$ acts faithfully on $P$. By Maschke's theorem, $P$ is the direct sum of irreducible $\mathrm{GF}(p)[Q]$-modules. Let $W \leqslant P$ be an irreducible $\operatorname{GF}(p)[Q]$-module. If $K$ is the kernel of $Q$ on $W$, then $Q / K$ is cyclic, and thus $K$ is a maximal subgroup of $Q$. Since the set of nonidentity elements of $P$ forms one automorphism orbit, it follows that, for each $1 \neq x \in P, C_{Q}(x)$ has index $q$ in $Q$. Also, the elements of order $q$ form one automorphism orbit, so that $\left|C_{P}(y)\right|=p^{c}$ for all $1 \neq y \in Q$ (for some $c \geqslant 0$ independent of $y$ ). Let $|P|=p^{a}$, 
and let $|Q|=q^{b}$. We now count the number of elements of $G$ of order $p q$. Note that if $w \in G$ has order $p q$, then $w=x y$, where $x$ has order $p, y$ has order $q$, and $x y=y x$; moreover, this representation is unique. Now each $1 \neq y \in Q$ has $|P| / p^{c}$ conjugates (which belong to distinct Sylow $q$-subgroups), and $y$ commutes with $p^{c}-1$ elements of order $p$. Hence the number of elements of order $p q$ is

$$
\left(q^{b}-1\right) p^{a}\left(p^{c}-1\right) / p^{c}=p^{a-c}\left(p^{c}-1\right)\left(q^{b}-1\right) .
$$

On the other hand, each $1 \neq x \in P$ commutes with the $\mid Q V / q=q^{b-1}$ elements of $Q$. Each of these except for the identity has $p^{a-c}$ conjugates (which belong to distinct Sylow $q$-subgroups). Hence the number of elements of order $p q$ is

$$
\left(p^{a}-1\right)\left(q^{b-1}-1\right) p^{a-c} \text {. }
$$

A comparison of the two counts yields

$$
\left(p^{c}-1\right)\left(q^{b}-1\right)=\left(p^{a}-1\right)\left(q^{b-1}-1\right) .
$$

If $b>1$, we thus have

$$
\left(p^{a}-1\right) /\left(p^{c}-1\right)=\left(q^{b}-1\right) /\left(q^{b-1}-1\right)=m, \text { say. }
$$

Now $q-1<m<q$, and $p^{a-c}-1<m<p^{a-c}$, so that $q-1<p^{a-c}<m+1$ $<q+1$. Thus $p^{a-c}=q$, which gives a contradiction. Hence $b=1$, as asserted. The proof is complete.

\section{References}

[1] B. Huppert, Endliche Gruppen I (Springer-Verlag, 1967).

[2] P. R. Sanders, 'The central automorphisms of a finite group', J. London Math. Soc. 44 (1969), 225-228.

[3] J. Walter, 'The characterization of finite groups with abelian Sylow 2-subgroups', Ann. of Math. 89 (1969), 405-514.

[4] D. Winter, 'The automorphism group of an extraspecial p-group', Rocky Mountain J. Math. 2 (1972), 159-168.

Department of Mathematics

University College

Dublin

Ireland
Department of Mathematics University College

Cork

Ireland 